\title{
Migrantenmedizin - ein wichtiges Thema für uns alle
}

Die klassische Definition der Tropenmedizin war, dass sie sich mit Parasitosen und mit Infektionen durch Erreger befasst, deren Entwicklung an die Bedingungen dieser Länder gebunden sind. In einem weiteren Sinn beschäftigt sich die Tropenmedizin aber mit allen in diesen Ländern vorkommenden Krankheiten und medizinischen Problemen, das heißt auch mit solchen, die in gemäßigteren Zonen vorkommen, dort aber eine geringere Häufigkeit haben, andere Verläufe zeigen oder nicht mehr stark von Bedeutung sind.

Tropenmedizin ist inhaltlich nicht statisch. Die positiven und negativen Entwicklungen in den Ländern der Tropen bedingen ständig wechselnde Anforderungen, zum Beispiel durch Urbanisierung, Bevölkerungswachstum, Kriege oder Veränderungen der Umwelt. Andererseits beschäftigt sich die Tropenmedizin mit Erkrankungen, die aus den Tropen in die Industrienationen importiert werden - oder genauer: mit den Menschen, die diese Erkrankungen mitbringen. Und das sind nun einmal Reisende und Migranten. Und Sie wissen genauso gut wie ich, dass es weltweit zu einer Zunahme der Reisenden, aber auch der Migrationsströme kommt.

In diesem Heft fasst A. Stich die Ergebnisse eines Workshops zur Gesundheitsversorgung von Migranten, der im März dieses Jahres in Würzburg stattgefunden hat, in einem Bericht (Seite 66-67) zusammen. Das Fazit ist: Migrantenmedizin ist ein ganz entscheidendes Thema für alle, die sich mit Tropenmedizin, Reisemedizin und verwandten Gebieten beschäftigen. Und deshalb wird Migrantenmedizin auch immer wieder ein wichtiges Thema für unsere Zeitschrift sein.

Gesundheitsprobleme von Migranten in Deutschland können importierte Erkrankungen aus dem Herkunftsland sein - Infektionskrankheiten, aber auch genetische Erkrankungen wie beispielsweise das familiäre Mittelmeerfieber. Denken Sie auch an genetisch be- dingte Unterschiede im Stoffwechsel: So ist bei der Therapie eines Hypertonus zu beachten, dass Afrikaner im allgemeinen niedrigere Plasmarenin- und Angiotensin-II-Spiegel haben als andere ethnische Gruppen.

Aber natürlich beinhaltet Migrantenmedizin noch viel mehr: Migration kann Einfluss haben auf das Risiko zu erkranken, aber auch auf die Chance, eine adäquate Therapie zu erhalten. Immigranten haben Kommunikationsprobleme. Dies hat Folgen für die Anamnese. Und das hat Konsequenzen für die Therapie: Stichwort Compliance. Wie kann jemand kooperieren, der wenig versteht? Aber nicht nur das, Krankheit ist - auch - ein kulturelles Konstrukt, das im Bedeutungssystem einer Kultur kodiert wird.

In unserer Gesellschaft ist die Grundlage hierfür die naturwissenschaftlich orientierte Medizin. Für Menschen anderer Kulturen kann Krankheit eine völlig andere Realität darstellen. Diese baut auf unterschiedlichen Krankheitstaxonomien und Nosologien auf. Ich glaube, wer Migranten aus den Tropen betreut, sollte hier einige grundlegende Kenntnisse besitzen (es kann nicht darum gehen, alle Kulturen zu verstehen - selbst Ethnologen haben wohl kaum diesen Anspruch). Deshalb ist ein Workshop, wie von Herrn Stich veranstaltet, so wichtig.
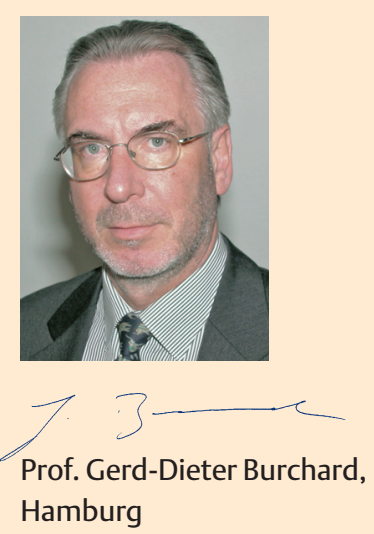